\title{
Plasmodium knowlesi: an update
}

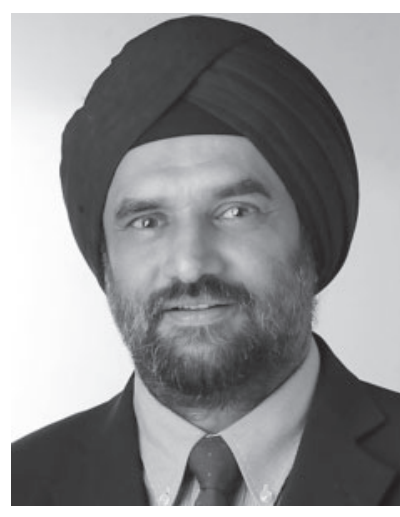

Balbir Singh

Malaria Research Centre University Malaysia Sarawak 94300 Kota Samarahan Sarawak, Malaysia Tel: +6082581000 Fax: +6082665152 Email: bsingh@unimas.my

There were only four species of Plasmodium that were thought to cause malaria in humans until a large number of human infections by Plasmodium knowlesi, a malaria parasite typically found in long-tailed and pig-tailed macaques, were reported in 2004 in Malaysian Borneo. Since then, cases of knowlesi malaria have been reported throughout South-east Asia and also in travellers returning from the region. This article describes the molecular, entomological and epidemiological data which indicate that $P$. knowlesi is an ancient parasite that is primarily zoonotic, and there are three highly divergent sub-populations. It also describes the detection methods for $P$. knowlesi, which is morphologicaly similar to $P$. malariae, and the clinical features and treatment of this malaria parasite that is potentially fatal.

\section{Malaria parasites and discovery of large focus of human knowlesi malaria cases}

Malaria is caused by parasites that belong to the genus Plasmodium and there are more than 150 species of Plasmodium that infect reptiles, birds and mammals ${ }^{1}$. These parasites, in general, tend to be host-specific. Long-tailed and pig-tailed macaques (Macaca fascicularis and $M$. nemestrina respectively) are hosts to five species (P. knowlesi, P. inui, P. cynomolgi, P. fieldi and P. coatneyi). Only four species of Plasmodium, namely P. falciparum, P. vivax, $P$. malariae and $P$. ovale, were thought to cause malaria in humans until a large number of human cases due to P. knowlesi were reported in Sarawak, Malaysian Borneo over 11 years ago ${ }^{2}$. The study in Kapit was prompted by observations that cases diagnosed by microscopy as $P$. malariae had high parasitaemias, required hospitalization and that $95 \%$ of patients were adults. This was in contrast to $P$. malariae infections which typically are asymptomatic with low parasitaemia and occur in all age groups. When blood samples from 208 malaria patients at Kapit Hospital were analysed by PCR assays, none were identified as $P$. malariae, although 141 had been diagnosed as $P$. malariae by microscopy. Fifty-eight percent (120) were either single $P$. knowlesi infections or mixed infections of $P$. knowlesi with $P$. falciparum and $P$. vivax. Misdiagnosis had occurred because the blood stages of $P$. knowles $i$ and P. malariae are morphologically indistinguishable ${ }^{3}$.

\section{Epidemiology and risk factors of acquiring knowlesi malaria}

Human infections with $P$. knowlesi have been reported throughout Malaysia and in Thailand, Singapore, the Philippines, Vietnam, Cambodia, Indonesia, Brunei, Myanmar and in the Nicobar and Andaman Islands of India ${ }^{4,5}$. In Malaysia, $P$. falciparum and $P$. vivax cases have declined over the past five years and $P$. knowlesi has now become the most common cause of human malaria ${ }^{6,7}$. The true incidence of knowlesi malaria is not known in other parts of Southeast Asia since not many large-scale studies have been undertaken with molecular detection assays.

The geographical distribution of human $P$. knowlesi infections is similar to that of the natural hosts of $P$. knowlesi, the long-tailed and pig-tailed macaques ${ }^{8}$. Reports from 1931 to 1970 identified macaques as hosts of $P$. knowlesi in Peninsular Malaysia, Singapore and the Philippines ${ }^{9}$, and a banded leaf monkey (Presbytis melalophos) in Peninsular Malaysia' ${ }^{9}$. Since 2007, P. knowlesi infections detected by molecular methods have been described in macaques in Peninsular Malaysia, Malaysian Borneo, Singapore and Thailand ${ }^{4}$.

The transmission of $P$. knowlesi in nature has been shown to be restricted to mosquitoes belonging to the Anopheles leucosphyrus group $^{10}$. The members of this forest-dwelling group of mosquitoes that have been identified as vectors include An. latens (in Sarawak, Malaysian Borneo) ${ }^{11}$, An. balabacensis balabacensis (in Sabah, Malaysian Borneo), An. dirus (in Vietnam) ${ }^{12}$ and An. hackeri and An. cracens (in Peninsular Malaysia) ${ }^{1,13}$.

People that are at risk of acquiring knowlesi malaria are those that enter the habitat of the macaque reservoir hosts and the Anopheline vectors at dusk or later as this coincides with the peak biting time of the vectors ${ }^{14,15}$. These include subsistence farmers, timber camp workers, hunters, army personnel and also travelers to forests or forest-fringe areas. Visitors to South-east Asia from Australia, USA, Finland, Sweden, Germany, France, New Zealand, Taiwan and Japan have acquired knowlesi malaria following holidays or working visits 
to Malaysian Borneo, Peninsular Malaysia, Brunei, Thailand, Indonesia and the Philippines ${ }^{16}$.

\section{Molecular and whole genome studies}

In order to understand the molecular epidemiology and demographic history of knowlesi malaria, the mitochondrial (mt) genome sequences of $P$. knowles $i$ were initially studied ${ }^{17}$. Certain mt haplotypes were shared between humans and macaques and there were no haplotypes that were associated exclusively with either host; further evidence supporting $P$. knowlesi as a zoonotic parasite. Additional analyses indicated that $P$. knowles $i$ was as old as, if not older than, $P$. falciparum and P. vivax, and that it underwent a population expansion between 30,000 to 40,000 years ago. Macaques colonized Asia over 5 million years ago ${ }^{18}$ and are probably the original hosts for P. knowlesi. A recent study, where 599 P. knowlesi samples from Peninsular Malaysia and Malaysian Borneo were analysed by a panel of ten microsatellite markers, showed there are two highly divergent sub-populations of $P$. knowlesi, and each of these subpopulations correspond with parasites from either longtailed or pig-tailed macaques ${ }^{19}$. More recently, genome-wide sequence analysis of clinical $P$. knowlesi isolates from Malaysian Borneo shows sub-population structure that matches the analysis using microsatellite markers and also demonstrate there is a third sub-population of parasites, corresponding to laboratory strains isolated over 50 years ago from Peninsular Malaysia and the Philippines $^{20}$. No signals of positive selection were observed in P. knowlesi around five orthologues of known P. falciparum drug resistance genes, indicating that the parasites in the reservoir macaque hosts have not been under antimalarial drug selection, thereby providing further evidence that knowlesi malaria is a zoonosis.

\section{Diagnosis}

In laboratories in malaria-endemic countries, malaria is diagnosed by examination of blood films by microscopy. Under the microscope, the early blood forms of $P$. knowlesi are identical to those of $P$. falciparum, while the other developmental stages, including the 'band forms', are similar to those of $P$. malariae ${ }^{3}$. There are minor morphological differences between these two species. The mature schizonts of $P$. knowlesi can contain up to 16 merozoites, whereas those of $P$. malariae have between $6-12^{3}$. However, mature schizonts are not found in all blood films examined and in diagnostic laboratories, where technologists are only trained to recognise $P$. falciparum, $P$. vivax, $P$. ovale and $P$. malariae, most $P$. knowlesi infections have been identified by microscopy as P. malariae ${ }^{2,4,21}$. Although morphologically similar, P. knowlesi parasites multiply every $24 \mathrm{~h}$ in the blood while this erythrocytic cycle is $72 \mathrm{~h}$ for $P$. malariae?

Molecular detection methods are the most sensitive and accurate techniques for identification of $P$. knowlesi. These include single and nested PCR assays, real-time PCR assays and loop-mediated isothermal assays ${ }^{4}$. However, these assays are relatively expensive, not rapid and are not readily available in resource-poor laboratories where the majority of $P$. knowlesi infections are detected. Rapid diagnostic tests (RDTs) for malaria are available, but the overall sensitivity of detection of a small number of RDTs that have been evaluated against knowlesi malaria cases varied between 26-74\% and was even lower (0-45\%) for parasitaemias below 1000 parasites/ $\mu \mathrm{L}^{22-24}$. Due to the rapid multiplication rate of $P$. knowlesi in the blood of $24 \mathrm{~h}$, sensitive RDTs capable of detecting knowlesi malaria at the early phase of infection are urgently required for rural laboratories.

\section{Clinical and laboratory features of knowlesi malaria}

P. knowlesi causes a wide spectrum of disease, from asymptomatic infections ${ }^{9,25}$ to fatal ones ${ }^{26-29}$. The most common presenting signs and symptoms reported are fever with chills, followed by headache, myalgia, poor appetite, arthralgia, cough, abdominal pain and diarrhoea $^{27}$. These are not significantly different to those observed in patients with vivax and falciparum malaria. The majority of cases $\left(93.5 \%{ }^{27}\right.$ and $\left.84.5 \%{ }^{30}\right)$ at district hospitals in Sarawak had uncomplicated malaria with a fatality rate of $2 \%$, whereas in a retrospective study in a referral hospital in Sabah, 61\% of 56 cases were uncomplicated and the fatality rate was $27 \%^{31}$. However, subsequently at the same referral hospital, the use of intravenous artesunate for severe malaria cases and artemisinin combination therapy for nonsevere cases, resulted in no deaths among 130 knowlesi malaria patients $^{29}$. Typical complications of severe knowlesi malaria in adults include jaundice, acute kidney injury, hypotension, acute respiratory distress syndrome and metabolic acidosis ${ }^{26,27,29,30,32}$. In adults, severe anaemia has not been observed and neither has cerebral malaria, while severe disease has not been noted in the relatively small number of children with knowlesi malaria ${ }^{4,33}$. Thrombocytopaenia is very common, occurring in 97.3 to $100 \%$ of knowlesi malaria patients, and together with parasitaemia, correlates with severity of disease $e^{27,30,31}$. Following a case control study, it was recommended that any patient with a platelet count of $<45000 / \mu \mathrm{L}$ or parasitaemia of $>35000$ parasites/ $\mu \mathrm{L}$ should be regarded at risk of developing complications and should be treated for severe malaria ${ }^{30}$. 


\section{Treatment of knowlesi malaria}

Since knowlesi malaria is primarily a zoonosis, the parasites have been under no antimalarial drug pressure and should be susceptible to all antimalarials. This has been observed in hospital-based studies as well as case reports where several antimalarials have been used successfully to treat knowlesi malaria patients ${ }^{4}$. P. knowlesi parasites are highly sensitive to chloroquine ${ }^{34}$ but following an informal consultation on the public health importance of knowlesi malaria organised by the WHO in 2011, it was recommended that in areas where knowlesi malaria has been detected, all infections diagnosed as $P$. malariae by microscopy should be treated and managed as for falciparum malaria ${ }^{35}$. Therefore, for uncomplicated knowlesi malaria cases in South-east Asia, artemisinin combination therapy is recommended. For severe knowlesi malaria, intravenous antimalarials should be administered and the use of artesunate in a tertiary referral hospital in Sabah was associated with zero mortality ${ }^{29}$.

\section{Future directions}

The available molecular, entomological and epidemiological data strongly indicate that knowlesi malaria is primarily a zoonosis. However, human-to-human transmission has been demonstrated under experimental conditions ${ }^{9}$ and it is not known whether it is currently occurring. The reasons for the increase in the number of knowlesi malaria cases, particularly in Malaysian Borneo, are also unknown. Whether the increase is due to increased awareness, changes in the feeding habits of the vectors, the destruction of the natural habitats of the macaque reservoir, human migration to areas close to macaque habitats, a recent adaptation of knowlesi malaria parasites to humans, or to some other factors needs to be investigated. In addition, currently available methods of control of human malaria involving the use of insecticide treated bednets and residual spraying of houses are ineffective against knowlesi malaria, where transmission primarily occurs outdoors. Therefore, effective methods of prevention and control need to be found and implemented, in order to prevent $P$. knowlesi from establishing itself in the human population.

\section{References}

1. Garnham, P.C.C. (1966) Malaria parasites and other haemosporidia. Blackwell Scientific Publications.

2. Singh, B. et al. (2004) A large focus of naturally acquired Plasmodium knowlesi infections in human beings. Lancet 363,1017-1024. doi:10.1016/S0140-6736(04) $15836-4$

3. Lee, K.S. et al. (2009) Morphological features and differential counts of Plasmodium knowlesi parasites in naturally acquired human infections. Malar. J. 8, 73. doi:10.1186/1475-2875-8-73

4. Singh, B. and Daneshvar, C. (2013) Human infections and detection of Plasmodium knowlesi. Clin. Microbiol. Rev. 26, 165-184. doi:10.1128/CMR.00079-12
5. Tyagi, R.K. et al. (2013) Discordance in drug resistance-associated mutation patterns in marker genes of Plasmodium falciparum and Plasmodium knowlesi during coinfections. J. Antimicrob. Chemother. 68, 1081-1088. doi:10.1093/jac/ dks 508

6. William, T. et al. (2013) Increasing incidence of Plasmodium knowlesi malaria following control of P. falciparum and P. vivax Malaria in Sabah, Malaysia. PLoS Negl. Trop. Dis. 7, e2026. doi:10.1371/journal.pntd.0002026

7. Yusof, R. et al. (2014) High proportion of knowlesi malaria in recent malaria cases in Malaysia. Malar. J. 13, 168. doi:10.1186/1475-2875-13-168

8. Cox-Singh, J. and Singh, B. (2008) Knowlesi malaria: newly emergent and of public health importance? Trends Parasitol. 24, 406-410. doi:10.1016/j.pt.2008.06.001

9. Coatney, G.R. et al. (1971) The primate malarias. US Department of Health, Education and Welfare.

10. Collins, W.E. (2012) Plasmodium knowlesi: a malaria parasite of monkeys and humans. Annu. Rev. Entomol. 57, 107-121. doi:10.1146/annurev-ento-121510133540

11. Vythilingam, I. et al. (2006) Natural transmission of Plasmodium knowlesi to humans by Anopheles latens in Sarawak, Malaysia. Trans. R. Soc. Trop. Med. Hyg. 100, 1087-1088. doi:10.1016/j.trstmh.2006.02.006

12. Marchand, R.P. et al. (2011) Co-infections of Plasmodium knowlesi, P. falciparum, and P. vivax among humans and Anopheles dirus mosquitoes, Southern Vietnam. Emerg. Infect. Dis. 17, 1232-1239. doi:10.3201/eid1707. 101551

13. Vythilingam, I. et al. (2008) Plasmodium knowlesi in humans, macaques and mosquitoes in peninsular Malaysia. Parasit. Vectors 1, 26. doi:10.1186/1756-3305$1-26$

14. Tan, C.H. et al. (2008) Bionomics of Anopheles latens in Kapit, Sarawak, Malaysian Borneo in relation to the transmission of zoonotic simian malaria parasite Plasmodium knowlesi. Malar. J. 7, 52. doi:10.1186/1475-2875-7-52

15. Vythilingam, I. (2012) Plasmodium knowlesi and Wuchereria bancrofti: their vectors and challenges for the future. Front. Physiol. 3, 115. doi:10.3389/ fphys.2012.00115

16. Cramer, J.P. (2015) Plasmodium knowlesi malaria: overview focussing on travelassociated infections. Curr. Infect. Dis. Rep. 17, 8. doi:10.1007/s11908-015-0469-6

17. Lee, K.S. et al. (2011) Plasmodium knowlesi: reservoir hosts and tracking the emergence in humans and macaques. PLoS Pathog. 7, e1002015. doi:10.1371/ journal.ppat.1002015

18. Ziegler, T. et al. (2007) Molecular phylogeny and evolutionary history of Southeast Asian macaques forming the M. silenus group. Mol. Phylogenet. Evol. 42, 807-816. doi:10.1016/j.ympev.2006.11.015

19. Divis, P.C. et al. (2015) Admixture in humans of two divergent Plasmodium knowlesi populations associated with different macaque host species. PLoS Pathog. 11, e1004888. doi:10.1371/journal.ppat.1004888

20. Assefa, S. et al. (2015) Population genomic structure and adaptation in the zoonotic malaria parasite Plasmodium knowlesi. Proc. Natl. Acad. Sci. USA 112, 13027-13032. doi: $10.1073 /$ pnas. 1509534112

21. William, T. et al. (2014) Changing epidemiology of malaria in Sabah, Malaysia: increasing incidence of Plasmodium knowlesi. Malar. J. 13, 390. doi:10.1186 1475-2875-13-390

22. Foster, D. et al. (2014) Evaluation of three rapid diagnostic tests for the detection of human infections with Plasmodium knowlesi. Malar. J. 13, 60. doi:10.1186/ 1475-2875-13-60

23. Barber, B.E. et al. (2013) Evaluation of the sensitivity of a pLDH-based and an aldolase-based rapid diagnostic test for diagnosis of uncomplicated and severe malaria caused by PCR-confirmed Plasmodium knowlesi, Plasmodium falciparum, and Plasmodium vivax. J. Clin. Microbiol. 51, 1118-1123. doi:10.1128 JCM.03285-12

24. Grigg, M.J. et al. (2014) Combining parasite lactate dehydrogenase-based and histidine-rich protein 2-based rapid tests to improve specificity for diagnosis of malaria due to Plasmodium knowlesi and other Plasmodium species in Sabah, Malaysia. J. Clin. Microbiol. 52, 2053-2060. doi:10.1128/JCM.00181-14

25. Fornace, K.M. et al. (2015) Asymptomatic and submicroscopic carriage of Plasmodium knowlesi malaria in household and community members of clinical cases in Sabah, Malaysia. J. Infect. Dis. doi:10.1093/infdis/iv475 
26. Cox-Singh, J. et al. (2008) Plasmodium knowlesi malaria in humans is widely distributed and potentially life threatening. Clin. Infect. Dis. 46, 165-171. doi: $10.1086 / 524888$

27. Daneshvar, C. et al. (2009) Clinical and laboratory features of human Plasmodium knowlesi infection. Clin. Infect. Dis. 49, 852-860. doi:10.1086 605439

28. Rajahram, G.S.etal. (2012) Deaths due to Plasmodium knowlesi malaria in Sabah, Malaysia: association with reporting as Plasmodium malariae and delayed parenteral artesunate. Malar. J. 11, 284. doi:10.1186/1475-2875-11-284

29. Barber, B.E. et al. (2013) A prospective comparative study of knowlesi, falciparum, and vivax malaria in Sabah, Malaysia: high proportion with severe disease from Plasmodium knowlesi and Plasmodium vivax but no mortality with early referral and artesunate therapy. Clin. Infect. Dis. 56, 383-397. doi: $10.1093 / \mathrm{cid} / \mathrm{cis} 902$

30. Willmann, M. et al. (2012) Laboratory markers of disease severity in Plasmodium knowlesi infection: a case control study. Malar. J. 11, 363. doi:10.1186/1475. 2875-11-363

31. William, T. et al. (2011) Severe Plasmodium knowlesi malaria in a tertiary care hospital, Sabah, Malaysia. Emerg. Infect. Dis. 17, 1248-1255. doi:10.3201/eid 1707.101017
32. Cox-Singh, J. et al. (2010) Severe malaria - a case of fatal Plasmodium knowlesi infection with post-mortem findings: a case report. Malar. J. 9, 10. doi:10.1186/ 1475-2875-9-10

33. Barber, B.E. et al. (2011) Plasmodium knowlesi malaria in children. Emerg. Infect Dis. 17, 814-820. doi:10.3201/eid1705.101489

34. Daneshvar, C. et al . (2010) Clinical and parasitological response to oral chloroquine and primaquine in uncomplicated human Plasmodium knowlesi infections. Malar. J. 9, 238. doi:10.1186/1475-2875-9-238

35. WHO (2011) Informal consultation on the public health importance of Plasmodium knowlesi. World Health Organization Regional Office for the Western Pacific Press.

\section{Biography}

Professor Balbir Singh is the Director of the Malaria Research Centre at University Malaysia Sarawak. His research interests include the epidemiology, pathogenesis and evolution of malaria parasites.

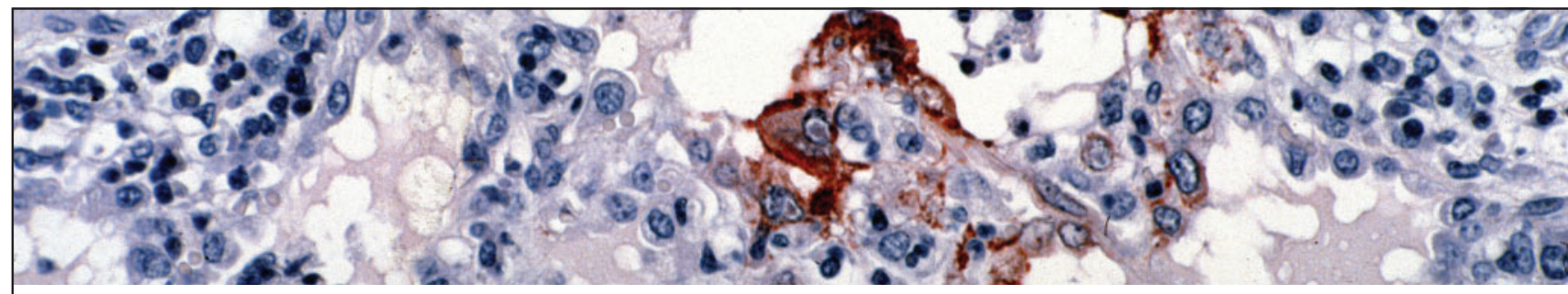

\section{Microbiology Australia}

Official Journal of the Australian Society for Microbiology Inc.

\section{Stay informed}

Keep up to date with industry news by subscribing to our email alerts or registering for RSS feeds.

www.publish.csiro.au/earlyalert
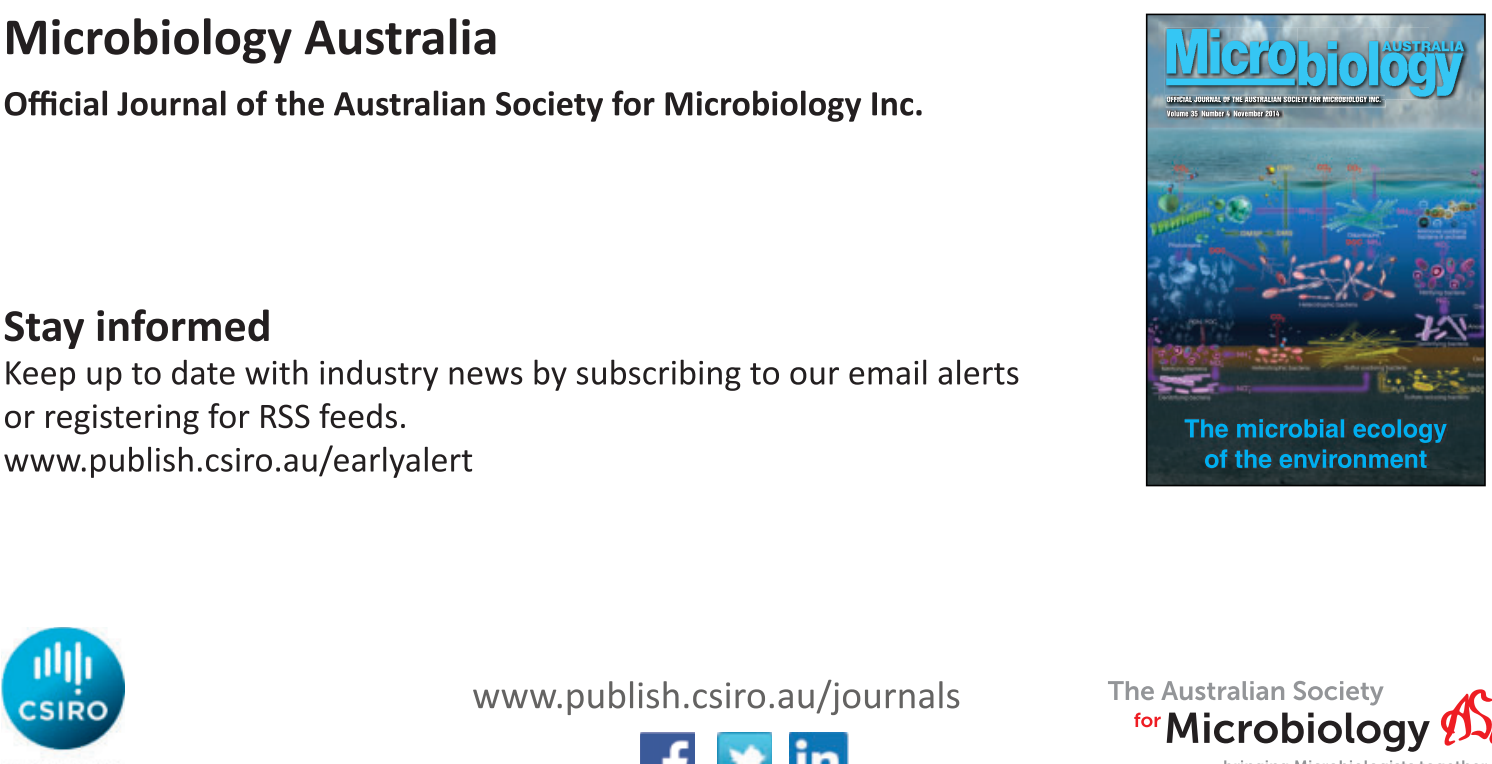

www.publish.csiro.au/journals 